
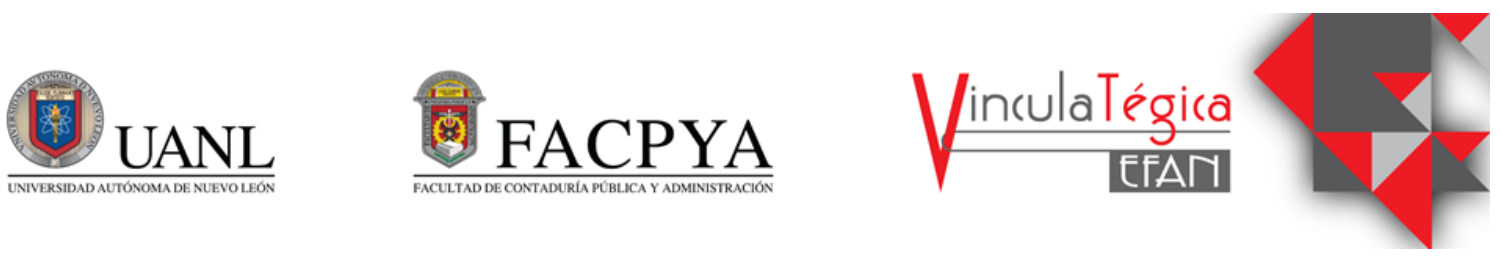

\title{
La Responsabilidad Social Universitaria un reto para la gestión educativa
}

\author{
Leonor Gutiérrez González ${ }^{1}$, Cesar Rolando Barboza Lara ${ }^{2}$ y Luis Horacio Salas Torres ${ }^{3}$ \\ ${ }^{1}$ Facultad de Contaduría y Administración UN Universidad Autónoma de Coahuila, \\ leonorgutierrez@uadec.edu.mx, Carretera 57,Km 4.5,866 6390760 \\ ${ }^{2}$ Facultad de Contaduría y Administración UN Universidad Autónoma de Coahuila, \\ cesar.barboza@uadec.edu.mx, Carretera 57,Km 4.5,866 6390760 \\ ${ }^{3}$ Facultad de Contaduría y Administración UN Universidad Autónoma de Coahuila, \\ luissalastorres@uadec.edu.mx, Carretera 57,Km 4.5,866 6390760
}

\begin{abstract}
Información del artículo revisado por pares
Fecha de aceptación: junio-2021

Fecha de publicación en línea: diciembre-2021

DOI: https://doi.org/10.29105/vtga7.2-61
\end{abstract}

\section{Resumen}

El trabajo de investigación describe la percepción que tienen los alumnos de la Facultad de Contaduría y Administración de la Universidad Autónoma de Coahuila, sobre los principales aspectos de la Responsabilidad Social Universitaria. Siendo de tipo descriptivo con enfoque cuantitativo y cualitativo. El objetivo principal fue analizar el conocimiento y participación de los estudiantes en los programas de apoyo que convocan el Gobierno y la Universidad. Dichos programas se orientaron en la problemática de los siguientes sectores de la población: pobreza, migrantes, ancianos, niños, jóvenes, desempleo, la mujer y caritas y/o Cruz Roja. Se realizó una muestra a 297 estudiantes de las carreras de Contaduría, Administración de Empresas y Administración de Recursos Humanos. Los primordiales hallazgos que resultaron de la investigación fue que existe un conocimiento impreciso sobre los procesos que convocan tanto el Gobierno como la Universidad hacia programas de apoyo a la sociedad, en cuanto a la participación de los universitarios, el resultado está considerado como "nunca" o "casi nunca". Se propone diseñar programas académicos que incorporen actividades dirigidas a reconocer $\mathrm{e}$ introducir eventos de acuerdo con las necesidades de la comunidad, apoyando en los problemas
The research work describes the perception that students of the Faculty of Accounting and Administration of the Autonomous University of Coahuila have, on the main aspects of University Social Responsibility. Being of a descriptive type with a quantitative and qualitative approach. The main objective was to analyze the knowledge and participation of the students in the support programs convened by the Government and the University.

Said programs were focused on the problems of the following population sectors: poverty, migrants, the elderly, children, youth, unemployment, women and caritas and / or the Red Cross. A sample was made of 297 students from the degrees of Accounting, Business Administration and Human Resources Administration. The main findings that resulted from the research was that there is imprecise knowledge about the processes that both the Government and the University call for programs to support society, in terms of the participation of university students, the result is considered "never" or "almost never. It is proposed to design academic programs that incorporate activities aimed at recognizing and introducing events according to the needs of the community, supporting the crucial problems of society. As well as, review the social service programs so that the purpose for which they were created is fulfilled. In this way, a socially responsible training in the university is presumed, which will have repercussions in the community in which you live and in the company where you work. 
cruciales de la sociedad. Así como, revisar los programas de servicio social para que se cumpla con el propósito para el cual fueron creados. De esta manera se presume una formación socialmente responsable en el universitario, que repercutirá en la comunidad en la que vive y en la empresa donde labore.

Palabras clave: Estudiantes Universitarios, Programas de apoyo, Responsabilidad Social Universitaria.

\section{INTRODUCCIÓN}

La responsabilidad social tiene sus orígenes desde la perspectiva empresarial, para luego verse incursionada en la universidad para la deliberación exhaustiva de su misión y la situación social en sus procesos claves: formación, investigación y extensión cultural. Mostrando cuatro impactos universitarios: organizacionales, educativos, cognitivos y sociales. Así lograr las destrezas de cambio organizacional y las labores que genera, orientadas a crear correlaciones entre los diferentes grupos de interés, internos y externos, que proyecten el desarrollo económico, social ambiental de la sociedad. (Vallaeys, De la Cruz, \& Sasia, 2009)

"La Responsabilidad Social Universitaria supera el enfoque de la proyección social y extensión universitaria y apuesta por una reflexión integral de la Universidad como institución académica que influye en el entorno social universitario". (Vallaeys et al., 2009) un campus responsable, implica la gestión socialmente responsable de la organización y de los procedimientos gubernamentales.

Bajo este panorama, el compromiso social de las instituciones educación superior (IES) aborda dos indicaciones principales; la primera describe la importancia en la validez del cumplimiento en las funciones, determinado como gestión de la calidad universitaria, y la segunda, tiene su afectación de una manera muy especial en el cumplimiento de las funciones en el marco de los requerimientos y de la dinámica activa de la sociedad, lo que se define como pertinencia. (Araque \& Peralta, 2016)

Justo es, concretar lo que se conoce por sociedad y por Universidad; para enseguida
Keywords: University Students, Support Programs, University Social Responsibility.

JEL Codes: I23, I31, I38

reflexionar la responsabilidad tacita que existe entre los dos sujetos de la relación, con la intención de explicar claramente el ámbito de la responsabilidad universitaria, en el marco de la pertinencia; y así finalmente, realizar las consideraciones oportunas sobre "lo social" como un aspecto que ha adquirido especial notabilidad en las posturas y circunstancias reales de dicha relación.

La Responsabilidad Social Universitaria, según lo considera el autor (Vallaeys, 2014), es una nueva actitud de gestión universitaria que se va desplegando en América Latina para responder a los atributos organizacionales y académicos de la universidad.

las Instituciones de educación superior, creadas para la generación del conocimiento y a la formación de profesionales (Vázquez, 2007) han determinado y definido su trabajo a partir de un conocimiento social, en algunas situaciones es orientada hacia el quehacer únicamente del conocimiento de manera exclusiva, por lo que fue calificada como "torre de marfil", pero a pesar de eso se ha edificado el conocimiento para la humanidad, avanzando en el tiempo con enfoques relacionados con la formación de los profesionales que requiere la sociedad, la réplica a las necesidades de la sociedad mediante la investigación y la extensión, el episodio político, la transferencia tecnológica, el desarrollo humano sostenible, entre otros conceptos que invariablemente actualizan día a día las formas en que la universidad ejerce su responsabilidad social de manera activa.

La Responsabilidad Social bajo la norma internacional ISO 26000: Para muchas organizaciones, la norma internacional ISO 26000 es un área que aún en estos tiempos es 
desconocido por las instituciones. Esta Guía de Responsabilidad Social (RS) fue publicada en noviembre del 2010, lo que resulta ya más de una década de su publicación, siendo el primer instrumento en la materia que fue construido con base en el consentimiento de un grupo multisectorial que contó con la colaboración de aproximadamente 450 expertos y 210 observadores de 99 países y 42 organizaciones internacionales, catalogados en seis categorías: industria, gobierno, consumidores, trabajadores, organizaciones no gubernamentales (ONG) y otros (principalmente universidades, servicios de consultoría e investigación y organismos de normalización y certificación). (Valencia, 2015)

Actualmente la universidad moderna en los últimos años ha experimentado una metamorfosis ubicándola en unos de los contextos más importantes, en el que la colectividad y la organización descubren como sentido fundamental al mantener sus métodos hacia el progreso. A partir de la instauración formativa resurgen programas y/o propósitos coligados a exploraciones que, mediante el establecimiento de adecuadas relaciones sociales y la corporación de nuevos programas y/o proyectos, que podrían apoyar a los sectores de la sociedad que requieran de su capital humano, de tal manera que exista una transformación en los medios, localizados a el aumento de productos de podrían beneficiar a la comunidad. (SALTOS \& VELAZQUEZ, 2020)

Continúan argumentando los autores, la Universidad intenta extenderse de manera que beneficie a su comunidad y esto es el método de acciones que le dan coyuntura a que realice su propósito en virtud de la vinculación con la confusa que vive el entorno y del compromiso primordial de los actores de la identidad universitaria a incidir positivamente en su entorno social, a partir de proyectos sociales, la apertura a proyectos diseñados para programas de educación continua y otras acciones de colaboración con empresas, instituciones gubernamentales y organismos en general, esto permitirá lograr lo que toda institución educativa prefiere, la excelencia en la calidad de sus programas y como consecuencia incrementar el estándar de los perfiles de egreso universitarios, suscitando un futuro profesional integro, por haber establecido una formación con la experiencia práctica de una realidad social, la cultura, las condiciones económicas, las organizaciones políticas y de poder, las normas y valores, las preferencias demográficas, de la comunidad.

\section{MARCO TEÓRICO}

La fundamentación de esta investigación se basa en diferentes documentos que apoyan la situación y abre el panorama acerca de la responsabilidad social en la Universidad desde el panorama de accionar de los estudiantes y su contribución en la formación ética.

El rol social de la universidad en Latinoamérica ha sido siempre objeto de muchos disputas y tensiones (Castañeda, Ruiz, Viloria, Castañeda, y Quevedo 2007; Boyle, 2004; Morles, 2004) y en la actualidad no escapa a la discusión. La masificación de la educación superior en Latinoamérica que desafía una mercantilización del mundo universitario y un proceso de internacionalización de la calidad sometida a codificaciones e indicadores de acreditación determinados desde Norteamérica y Europa, en argumentos sociales y económicos desiguales y diferentes. (Vallaeys \& Álvarez Rodríguez, 2019) además, argumentan los autores: La Responsabilidad Social Universitaria (en adelante RSU) es una política de gestión universitaria, que según Vallaeys (2014) se conjuga como una perfeccionamiento del concepto de Responsabilidad Social. Esta área ha provocado el interés de estudiosos del tema en los últimos tiempos; cada uno de estos investigadores tienen posturas diferentes e interesantes después de haber realizado numerosas fuentes del conocimiento. sobre el tema (De la Calle y Giménez, 2011; LarránJorge y Andrades- Peña, 2017; Mazzetti, 2014; Pacenza y Silva, 2013), no se ha realizado un estudio metodológico a mostrar el progreso de este concepto, accediendo a identificar de manera oportuna los documentos y autores principales, y que presente matices relevantes. 
Recientemente se observa que enunciado de responsabilidad social universitaria (RSU) indica de manera precisa la observancia de la importancia que existe en la universidad dentro de unos patrones y/o mediciones éticas, enfocados en la empatía por una sociedad, respecto a lo que viven sus habitantes y como se sostiene al medio ambiente en el que habita, así como al ambiente que provoca. De esta manera, la intención de la institución universitaria no solo tendrá como objetivos la impartición de cátedras de formación profesional, tendrá además que preocuparse por influir en sus alumnos con prácticas que generen principios éticos que les permitan comportarse como buenos ciudadanos e involucrarse en la solución de los problemas de la comunidad que cada vez carece de las necesidades primarias. (Erazo-Coronado, LlanoAristizábal, Garcés-Prettel, \& ErazoCoronado, 2020)

A lo largo del tiempo, la universidad ha rescatado una enorme función como cauce de la instauración y transmisión del conocimiento a la vez que es un eslabón entre la sociedad y las organizaciones económicas, los gobiernos públicos o las instituciones sociales.

Dentro del patrón socioeconómico que se ha ido perfeccionando en el cambio de siglo, el conocimiento ha dejado de ser la columna primordial para la creación de riqueza, el progreso sostenible, el fomento del paralelismo de oportunidades y la introducción de nuevas organizaciones de participación de la ciudad en la adopción de decisiones. Menciona el autor (de la Cuesta González, de la Cruz Ayuso, \& Fernández, 2010)

Es conocido y obviamente aceptable que la RSU estuviera como tema principal en la última Conferencia Mundial sobre la Educación Superior realizada por la UNESCO (5 al 8 de julio de 2009), teniendo como fundamentación: "Las instituciones de Educación Superior en su responsabilidad social tiene la obligación de avanzar en la penetración de la complejidad desafiante y global de manera presente y futura y en motivar hacia el descubrimiento de contenidos que fomenten conocimientos y acciones para realizar proyectos destinados beneficio social. UNESCO 2010 Pag.48 (Calderón, Pedro, \& Vargas, 2011).

Siendo así, lo mejor es establecer mecanismos que hagan posible pensar que la metodología a realizar para establecer la responsabilidad social universitaria, de acuerdo con lo expresado por (Ramos, De la Calle Maldonado, Martínez, \& De Dios Alija, 2016), es de los comentarios y argumentos más adecuados en relación con los valores éticos que componen y fomentan el diseño de responsabilidad generacional que emerge de la institución educativa hacia los compromisos sociales situados con los más necesitados.

Lo anterior logra concebir que, en los organismos que son creadores, los más importantes mandos que planifican, organizan y dirigen los métodos que deberán utilizarse estratégica y prácticamente deben incumbir de manera formal y comprometida de tal forma que se establezcan e implementen inteligentemente la base para el desempeño en el desarrollo de las habilidades propias y consignadas al programa destinado a mejorar una situación específica de la sociedad.

La universidad pública se ha enfocado por atender indicadores de calidad desde inicios de los noventa (Rubio Oca, 2006) que manifiesten a políticas públicas que ubican a las universidades en función de las peticiones del mercado de trabajo y su contribución a la productividad económica. Es claro que la cuestión del capital humano ha tenido gran dominio en la intrepidez de las políticas educativas de las universidades públicas desde el periodo de los ochenta. (Flores Crespo, 2005).

Será un reto para nuestro siglo XXI crear una procedencia social, donde la responsabilidad social de las universidades públicas en el transcurso de formación residente adquiere un enorme protagonismo. (Gasca-Pliego \& OlveraGarcía, 2011).

La Responsabilidad Social Universitaria (RSU) es habitualmente un concepto joven que extiende el análisis, el desarrollo y la reflexión del mundo empresarial en cuanto a las contribuciones y beneficios que una organización puede 
prometer a una sociedad. Sin embargo, la idea institucional requiere ir más allá de la filantropía y la generosidad que pueda ofrecer la universidad a un sector necesitado de la sociedad; más bien, esta percepción puede entenderse como la especulación y la observación que una institución educativa hace frente a su propia Misión, en términos de las contribuciones a su medio ambiente. (Arango Tobón, Clavijo Zapata, Puerta Lopera, \& Sánchez Duque, 2014), asociado con lo anterior, se menciona que "los conocimientos sobre las variables psicológicas que incidirían en ser socialmente responsable, permitiendo atender desde las Universidades el impacto de la educación superior en la formación de profesionales bien cualificados y con alta consideración a su deber con la sociedad, tal y como indican las directrices de la UNESCO y las competencias que identifica la OECD”. (Noguera, 2011)

Así mismo, el autor menciona "las Universidades no podían quedarse alejadas de la reflexión sobre Responsabilidad Social, ellas también son organizaciones, que a través de sus principales propósitos: formación humana y profesional (propósito académico) y construcción de nuevos conocimientos (propósito de investigación) tienen impactos específicos distintos a los generados por las empresas". (Domínguez Pachón, 2012)

Los autores argumentan; que La Responsabilidad Social Universitaria (RSU) "no se trata solo de una visión de asistencia social sino de una visión de conciencia social desde la perspectiva de un sentimiento unificado de toda la comunidad universitaria, que se interesa en disminuir los problemas sociales contribuyendo directamente con el aporte de capital social y humano, altamente competente para el desarrollo de la sociedad". (Ayala, 2012:29). La RSU es parte de la ética de la sociedad universitaria (estudiantes, docentes y personal administrativo), a través de la gestión responsable de los impactos educativos, cognitivos, profesionales, sociales y ambientales que la Universidad compone (François Vallaeys, 2005), en un dialogo participativo con la sociedad, para promover el Desarrollo Humano razonable. Percibe todos los contornos de la faena universitaria: docencia, investigación, y extensión universitaria y a todos los integrantes de la comunidad universitaria. (Paula \& Reyes, 2019)

El enfoque que se tomara es la responsabilidad social en las universidades donde como antecedente se puede partir según la UNESCO (1998) del patrimonio del conocimiento y la sociedad de la información que desarrollan la responsabilidad social de las universidades en proporción de la producción y propagación del conocimiento generado en su interior, lo que exige a estas instituciones a proporcionar más atención a las peticiones y requerimientos de la sociedad respecto de su quehacer, evolucionando en uno de los primeros referencias formales de la diligencia de la RSU al ámbito universitario.

(Ojeda-Hidalgo, Trapala-Sánchez, \& PazMarcano, 2020)

Enmarcando estudios realizado en relación al tema, la investigación de (Alférez Villrreal, 2014), en su exploración sobre: La colaboración social curricular en la responsabilidad social universitaria: El caso de la Facultad de educación de la universidad Internacional de Catalunya. Basada bajos el enfoque gerencial, transformacional y normativo. $\mathrm{La}$ investigación es positivista, interpretativo y hermenéutico, con diseño cuasi experimental en un estudio de caso, con métodos, técnicas e instrumentos mixtos. La conclusión arribada fue que medir la RSU en todas sus formas son complejas ya que existen elementos invisibles que interfieren o sesgan la investigación, pero la aproximación encontrada que hay participación curricular social de forma heterogénea.

(Lagunas, Ramírez, \& Rodríguez, 2017) realizaron una investigación titulada: "Un análisis de la percepción que los gerentes y profesores tienen de la RSU en las facultades de contabilidad y administración en México". Entre sus desenlaces se indican: Estos son funcionarios de mayor peso en la alineación y perfeccionamiento de las competencias en los estudiantes, primariamente el fortalecimiento de sus compendios, y de definición e interacción en la sociedad con sentido de responsabilidad. La 
inconstante organizacional, se influye a la no descomposición, transparencia y acato de cuentas en todos los métodos de gestión. Se observa que, en las carreras estudiadas, existen varios factores de gran notabilidad para la percepción del RSU, entre las cuales se destacan la formación educativa y organizacional.

La política que gestiona a la universidad con su comunidad, de forma democrática, imparcial, transparente, sostenible y ética, velando el clima y ambiente laboral, la gestión del talento humano, la gestión de residuos e impactos medioambientales producida por la universidad. Muestra además, el autor (Tiza, Huamancaja, \& Alva, 2020) menciona que la gestión académica refiere a que la universidad es la responsable de elaborar su currículo institucional con participación de los grupos de interés, bajo el rigor científico, académico, tecnológico y con directrices vigentes, atendiendo las demandas y necesidades de su entorno.

\section{MÉTODO}

La acometida del proyecto de investigación inicia evaluando la percepción de 45 estudiantes de la materia de Responsabilidad Social de las Organizaciones, construyendo propuestas enfocadas hacia los siguientes sectores donde más se requiere el apoyo y se determinaron los siguientes: pobres, migrantes, ancianos, niños, jóvenes con adicciones, desempleo y la mujer. De acuerdo con este estudio, se realiza un instrumento que muestra, si existe o no, el interés de apoyo por parte de las instituciones de gobierno, asociaciones civiles, organismos no gubernamentales, etc. Además de incluir que tanto hay participación por parte de los universitarios en los programas del gobierno y de la universidad.

La investigación es de tipo exploratorio-descriptivo con enfoque cuantitativo a través de una encuesta aplicada a una muestra de 297 estudiantes de la Facultad de Contaduría y Administración Unidad Norte de la Universidad Autónoma de Coahuila, utilizando para su forms

El procedimiento para calcular la confiabilidad del instrumento fue el método de formas alternativas o paralelas (Hernández, 2006) a partir de la aplicación del instrumento final a tres docentes investigadores y treinta estudiantes en el cual se comparó la similitud de los diferentes ítems en el instrumento aplicado. El resultado fue de .891 siendo una alta y positiva correlación entre los resultados por lo cual se aceptó la confiablidad de este.

El proceso de análisis de información se realizó a través del software SPSS 22.0 que proporciona cálculo de frecuencia y porcentaje.

Se consideran a los estudiantes que cursan diferentes carreras y semestres de la Facultad de Contaduría y Administración de la Universidad Autónoma de Coahuila, ubicada en la ciudad de Monclova, Coahuila, como unidad de análisis y las cuales constituyen el universo; el estudio se efectuó con un enfoque cuantitativo y se hizo una encuesta con una muestra de 297 estudiantes, de una población de 1289, según datos proporcionados por la Institución en cuestión. se utilizó una escala de Likert, en donde el 1 era $\mathrm{N}$ "Nunca", 2 CN "Casi Nunca", 3 AV "A Veces", 4 CS "Casi Siempre" y el $5 \mathrm{~S}$ "Siempre".

El contenido del cuestionario consta de lo siguiente: primera sección, siete preguntas generales; segunda sección: Programas de apoyo del Gobierno dirigidas a la sociedad, tercera sección: la participación del estudiante en actividades de voluntariado del Gobierno, cuarta sección: Programas de apoyo de la Universidad dirigidas a la sociedad y quinta sección: la participación del estudiante en las actividades de apoyo de la Facultad a la sociedad. Cada una de las secciones se enfocan en los sectores de apoyo que identificaron los alumnos de la materia de Responsabilidad Social de las Organizaciones y que en base a este estudio previo se realizó el estudio.

\section{RESULTADOS}

El cuestionario lo respondieron 233 mujeres y 64 hombres, donde el $80 \%$ son de la carrera de Contaduría, el $12 \%$ de Administración de empresas y el 8\% de Administración de Recursos Humanos. Siendo estudiantes de IV al VIII semestre, contestado además las siguientes preguntas: si conocen el termino Responsabilidad Social de las Organizaciones, 
son 220 quienes, si lo identifican, el resto no. A la pregunta sobre si conocen del término, Responsabilidad Social Universitaria, fueron 153 estudiantes que si saben a qué se refiere y el resto no han tenido oportunidad de conocer sobre el término.

Además, se cuestionó sobre: si consideraba que actualmente existe en su entorno una situación económica difícil, 218 contestaron de manera positiva, y la última pregunta, es si consideran que hay un problema de adicciones en los jóvenes, estando 234 de acuerdo en esta problemática.

A continuación, se muestran las tablas que contienen los resultados y que están determinadas, primero, lo que corresponde a los Programas del Gobierno en apoyo a la sociedad, luego, participación del estudiante en esos programas del gobierno, enseguida, los programas de apoyo de la Universidad y, la participación de los estudiantes en esos programas de apoyo que convoca la universidad.

Tabla No. 1

Sectores a donde van dirigidos los apoyos de los programas del gobierno

\begin{tabular}{|l|r|r|r|c|}
\hline \multirow{2}{*}{ Programas } & \multicolumn{1}{c|}{$\mathrm{N}$} & \multicolumn{1}{c|}{ Mín } & \multicolumn{1}{c|}{ Máx } & Media \\
\hline Pobreza & 297 & 1.00 & 5.00 & 2.6094 \\
\hline Migrantes & 297 & 1.00 & 5.00 & 2.0303 \\
\hline Ancianos & 297 & 1.00 & 5.00 & 3.3872 \\
\hline Niños & 297 & 1.00 & 5.00 & 2.7104 \\
\hline Jóvenes & 297 & 1.00 & 5.00 & 2.2458 \\
\hline Desempleados & 297 & 1.00 & 5.00 & 2.6162 \\
\hline Mujer & 297 & 1.00 & 5.00 & 3.0539 \\
\hline \multicolumn{4}{|c|}{ Fuente: elaboración propia } \\
\hline
\end{tabular}

Según la percepción de los encuestados, los apoyos del gobierno están dirigidos principalmente a los sectores del asilo de ancianos que tiene la media de 3.38 y le sigue el apoyo a la mujer con una media de 3.05 , lo más bajo se considera el sector de apoyo a migrantes con 2.03, a los jóvenes con 2.24 , a los más pobres con 2.60, para los desempleados $2.61 \mathrm{y}$, por último, para los más pobres 2.60
Tabla No. 2

Resultado de la participación de los estudiantes en programas de apoyo del Gobierno

\begin{tabular}{|l|r|r|r|r|}
\hline $\begin{array}{c}\text { Participación en } \\
\text { programas }\end{array}$ & \multicolumn{1}{c|}{$\mathrm{N}$} & \multicolumn{1}{c|}{ Mín } & \multicolumn{1}{c|}{ Máx } & Media \\
\hline Camp alimen & 297 & 1.00 & 5.00 & 3.1347 \\
\hline Migrantes & 297 & 1.00 & 5.00 & 2.0943 \\
\hline Asilo ancianos & 297 & 1.00 & 5.00 & 2.7744 \\
\hline Casa hogar & 297 & 1.00 & 5.00 & 2.6364 \\
\hline Prev adicciones & 297 & 1.00 & 5.00 & 2.0606 \\
\hline Prog emprend & 297 & 1.00 & 5.00 & 2.1380 \\
\hline Mujer & 297 & 1.00 & 5.00 & 2.5185 \\
\hline Caritas y/o CR & 297 & 1.00 & 5.00 & 3.0539 \\
\hline \multicolumn{5}{|c|}{ Fuente: elaboración propia } \\
\hline
\end{tabular}

La tabla No.2 muestra en que programas de Gobierno han participado los estudiantes, la mayoría de los resultados están debajo de la media, salvo en lo que se refiere a campaña de alimentos con una media de 3.13 y Caritas y/o Cruz Roja con una media de 3.05. los demás sectores muestran: migrantes, 2.09, apoyo a los ancianos 2.77, a los niños de la casa hogar 2.63, prevención de adicciones 2.06, capacitación a emprendedores 2.13 , a la mujer 2.15. Siendo de esta manera casi nulo el apoyo a los sectores que se mencionan.

Tabla No. 3

Sectores a donde van dirigidos los apoyos de los programas de la Universidad

\begin{tabular}{|l|c|r|r|r|}
\hline $\begin{array}{c}\text { Programas de la } \\
\text { Universidad }\end{array}$ & $\mathrm{N}$ & \multicolumn{1}{c|}{ Mín } & \multicolumn{1}{c|}{ Máx } & \multicolumn{1}{c|}{ Media } \\
\hline Camp alimen & 297 & 1.00 & 5.00 & 3.2761 \\
\hline Migrantes & 297 & 1.00 & 5.00 & 1.8788 \\
\hline A. Ancianos & 297 & 1.00 & 5.00 & 2.9192 \\
\hline Casa hogar & 297 & 1.00 & 5.00 & 2.8081 \\
\hline Pre.adicciones & 297 & 1.00 & 5.00 & 2.1010 \\
\hline Prog emprend & 297 & 1.00 & 5.00 & 2.8047 \\
\hline Mujer & 297 & 1.00 & 5.00 & 2.4377 \\
\hline Caritas CR & 297 & 1.00 & 5.00 & 2.8552 \\
\hline \multicolumn{4}{r|}{ Fuente: elaboración propia } \\
\end{tabular}

En cuanto a la tabla No. 3, que muestra los programas que la Universidad en algún 
momento ha convocado a apoyar, se observa que únicamente la "campaña de alimentos" tiene una media de 3.27. lo que significa que en algunas ocasiones se ha apoyado, para los demás programas las medias son muy bajas.

Siendo el apoyo a los migrantes el más bajo de 1.87, luego la prevención de adicciones con 2.10. Por lo que se presume que son pocos los programas en los que se trabajan con beneficio a la sociedad.

Tabla No. 4

Resultado de la participación de los estudiantes en programas de apoyo de la Universidad

\begin{tabular}{|l|r|r|r|r|}
\hline $\begin{array}{l}\text { Participación en } \\
\text { programas de la } \\
\text { Universidad }\end{array}$ & \multicolumn{1}{c|}{$\mathrm{N}$} & \multicolumn{1}{c|}{ Mín } & \multicolumn{1}{c|}{ Máx } & \multicolumn{1}{c|}{ Media } \\
\hline Camp alimen & 297 & 1.00 & 5.00 & 2.7273 \\
\hline Migrantes & 297 & 1.00 & 5.00 & 2.0034 \\
\hline A.ancianos & 297 & 1.00 & 5.00 & 2.6229 \\
\hline Casa hogar & 297 & 1.00 & 5.00 & 2.6633 \\
\hline Pre adiccion & 297 & 1.00 & 5.00 & 1.2828 \\
\hline Prog emprend & 297 & 1.00 & 5.00 & 2.3131 \\
\hline Mujer & 297 & 1.00 & 5.00 & 2.5522 \\
\hline Caritas CR & 297 & 1.00 & 5.00 & 3.8552 \\
\hline \multicolumn{5}{|r|}{ Fuente: elaboración propia } \\
\end{tabular}

La tabla 4, muestra lo referente a la participación de los estudiantes en los programas de apoyo que convoca la universidad, como se observa, casi en la mayoría de los diferentes sectores muestran una media menor a 3. Solo el sector de Caritas/Cruz Roja se tiene un resultado de 3.85 . en cuanto a las demás medias, en lo que se refiere a campaña de alimentos es 2.72, migrantes 2.00, para el asilo de ancianos 2.62, la casa hogar (niños) 2.66, prevención de adicciones 1.28, programa de emprendedores 2.31 , y apoyo a la mujer 2.55

\section{CONCLUSIONES}

De acuerdo con los resultados obtenidos se puede concluir que existe un desafío en cuanto corresponde a fomentar en los universitarios a tener una participación activa y comprometida para realizar actividades altruistas y de servicio para los sectores que más lo requieran.

Por tanto, se propone diseñar programas académicos que incorporen actividades dirigidas a reconocer e impactar en las escaseces de la comunidad, apoyando en los problemas cruciales de su sociedad.

Ya que en los resultados se identificó que existe una diferencia significativa entre el conocimiento y la colaboración de los estudiantes en los esquemas de apoyo que convoca el Gobierno y los de la Universidad.

La visión que existe en el ámbito de los estudiantes que respondieron el cuestionario accede a concebir que el tema investigado cuenta con restricciones en su establecimiento, al instituir una considerada distribución en las técnicas inherentes, actividades que se pueden realizar dentro de la investigación, y que en ocasiones se ha ignorado tanto por los docentes como por los estudiantes, por tanto, se propone que se realicen proyectos enfocados al apoyo a la comunidad, mismos que estén basados en el diseño de una metodología de investigación.

Es elemental concebir que, para quienes formamos parte de la educación universitaria debería de existir un interés en los temas que sumen actividades que crean y fomenten el conocimiento e instrucción, relacionada con la realización de cursos y/o talleres, de acuerdo con las necesidades del sector, si esto se logra a través de los estudiantes con la coordinación del docente, sería un avance muy significativo si se logra su establecimiento, puesto que se puede especular que no es de utilidad su aplicación, sin embargo, la sensibilización hacia la enseñanza de alguna disciplina y a los valores morales; son cada vez más importantes en la sociedad

Mediante este establecimiento y configuración, la gestión educativa tiene el reto de estar en el impulso de crear proyectos que impliquen temas orientados a convocar acciones derivadas de vínculos que propicien actividades desarrolladas en situaciones que requieran de mejorar la sociedad y los individuos, la capacitación en el emprendimiento, la prevención de 
adicciones, entre otros.

Se concluye también de acuerdo con los resultados obtenidos que la muestra seleccionada, desconoce de la existencia de programas de apoyo por parte del Gobierno y por parte de la Universidad. así mismo, el resultado de la muestra en cuanto a la participación en algunos programas, resulta por demás decadente.

La experiencia de este estudio nos permitió participar en una convocatoria con un proyecto en apoyo a los jóvenes que habitan en las colonias urbano marginadas de Monclova, Coahuila, con el objetivo de formarlos social y laboralmente, esto con el apoyo de estudiantes de la Facultad de Contaduría y Administración, lo que nos permite contribuir en el tratamiento del tema de Responsabilidad Social Universitaria.

Los datos obtenidos nos establecer como reto educativo la oportunidad de crear y establecer programas y/o proyectos dirigidos a apoyar a una sociedad que tiene identificadas sus carencias que pudieran ser motivo de solucionar por las instituciones de educación superior y de esta manera lograr beneficios mutuos; por un lado, el compromiso por parte de los involucrados de crear programas que contribuyan al bien común y que sean realizados por la comunidad universitaria; y, por otro lado, una sociedad satisfecha de los bienes recibidos.

El compromiso de la universidad ante la sociedad con este tipo de apoyos lograra alcanzar metas que actualmente son exigidas por instituciones nacionales e internacionales. 


\section{REFERENCIAS}

Alférez Villrreal, A. (2014). La participación social curricular en la Responsabilidad Social Universitaria: El caso de la Facultad de Educación de la Universitat Internacional de Catalunya. Universitat Internacional de Catalunya.

Arango Tobón, O. E., Clavijo Zapata, S. J., Puerta Lopera, I. C., \& Sánchez Duque, J. W. (2014). Formación académica, valores, empatía y comportamientos socialmente responsables en estudiantes universitarios. Revista de la educación superior, 43(169), 89-105.

Araque, Y. V. C., \& Peralta, C. M. P. (2016). La Responsabilidad Social Universitaria: emprendimiento sostenible como impacto de intervención en comunidades vulnerables. Revista Escuela de Administración de Negocios(81), 1-19.

Calderón, A. I., Pedro, R. F., \& Vargas, M. C. (2011). Responsabilidad social de la Educación Superior: la metamorfosis del discurso de la UNESCO en foco. Interface-Comunicação, Saúde, Educação, 15(39), 1185-1198.

de la Cuesta González, M., de la Cruz Ayuso, C., \& Fernández, J. M. R. (2010). Responsabilidad social universitaria: Netbiblo.

Domínguez Pachón, M. J. (2012). Responsabilidad social universitaria.

Erazo-Coronado, L., Llano-Aristizábal, S., Garcés-Prettel, M., \& Erazo-Coronado, A.-M. (2020). Impacto de la comunicación de la responsabilidad social universitaria en la selección de universidad y efecto mediador de la reputación. Profesional de la Información, 29(4).

Flores Crespo, P. P. F. C. (2005). Educación superior y desarrollo humano: el caso de tres universidades tecnológicas.

Gasca-Pliego, E., \& Olvera-García, J. C. (2011). Construir ciudadanía desde las universidades, responsabilidad social universitaria y desafíos ante el siglo XXI. Convergencia, 18(56), 3758.

Hernández, S. R. F., Collado Carlos; Baptista, Lucio Pilar. (2006). Metodología de la investigación (4a ed., Vol. 4).

Lagunas, E. A., Ramírez, D. M., \& Rodríguez, J. O. (2017). Un análisis sobre la percepción que los directivos y docentes tienen de la responsabilidad social universitaria en las facultades de contaduría y administración en México. Revista Universidad y Empresa, 19(32), 37-59.

Noguera, J. J. M. (2011). Responsabilidad social universitaria: estudio acerca de los comportamientos, los valores y la empatía en estudiantes de Universidades iberoamericanas. Universitat de València.

Ojeda-Hidalgo, J. F., Trapala-Sánchez, S. Y., \& Paz-Marcano, A. I. (2020). Responsabilidad social en las universidades. Revista Relayn-Micro y Pequeñas empresas en Latinoamérica, 4(2), 113-130.

Paula, I. A. N., \& Reyes, E. B. (2019). Valores, Responsabilidad Social Universitaria (RSU), Educación para el Desarrollo Sostenible (EDS) y Gestión de la Comunicación Organizacional. GECONTEC: Revista Internacional de Gestión del Conocimiento y la Tecnología, 7(1), 104-115.

Ramos, J. M. G., De la Calle Maldonado, C., Martínez, M. C. V., \& De Dios Alija, T. (2016). Hacia la validación del constructo" responsabilidad social del estudiante universitario"(RSEU). Bordón. Revista de pedagogía, 68(3), 41-58.

Rubio Oca, J. (2006). La política educativa y la educación superior en México, 1995-2006: un balance.

SALTOS, M. A., \& VELAZQUEZ, R. M. (2020). La responsabilidad social universitaria desde la perspectiva de los universitarios de Guayaquil. Revista ESPACIOS, 4l(18).

Tiza, D. O. H., Huamancaja, R. M. T., \& Alva, W. A. R. (2020). Responsabilidad social universitaria en dos facultades de la Universidad Nacional del Centro del Perú-2019. Ciencia Latina Revista Científica Multidisciplinar, 4(2), 286-297.

Valencia, W. A. (2015). La responsabilidad social: análisis del enfoque de ISO 26000. Industrial data, 18(2), 55-60. 
Vallaeys, F. (2014). La responsabilidad social universitaria: un nuevo modelo universitario contra la mercantilización. Revista iberoamericana de educación superior, 5(12), 105-117.

Vallaeys, F., \& Álvarez Rodríguez, J. (2019). HACIA UNA DEFINICIÓN LATINOAMERICANA DE RESPONSABILIDAD SOCIAL UNIVERSITARIA. APROXIMACIÓN A LAS PREFERENCIAS CONCEPTUALES DE LOS UNIVERSITARIOS. Educacion XX1, 22(1). Vallaeys, F., De la Cruz, C., \& Sasia, P. M. (2009). Responsabilidad social universitaria: manual de primeros pasos: Inter-American Development Bank.

Vázquez, S. G. (2007). Responsabilidad Social Universitaria "Acerca del desafío de formar profesionales con vocación y compromiso comunitario". Red Iberoamericana de Universidades por la Responsabilidad Social, Atenea, Universidad de la Marina Mercante, Argentina. 